\title{
Influence of weather conditions in the Yakutsk array region on Cherenkov light flux measurements
}

\author{
Igor Petrov ${ }^{* \dagger}$ \\ Yu.G. Shafer Institute of Cosmophysical Research and Aeronomy \\ E-mail: igor.petroveikfia.ysn.ru

\section{Stanislav Knurenko} \\ Yu.G. Shafer Institute of Cosmophysical Research and Aeronomy \\ E-mail: knurenko@ikfia.ysn.ru
}

\begin{abstract}
A comparison is made of the calculation of the spatial distribution of Cherenkov radiation from the primary proton performed with allowance for aerosol attenuation, with experimental data measured under different weather conditions at the Yakutsk EAS installation. It is shown that the transparency of the atmosphere affects the shape of the radial distribution of Cherenkov photons. With visibility range $\mathrm{V}=15 \mathrm{~km}$, calculations are close to experimental measurements, and more than $25 \mathrm{~km}$ - are minimal. This circumstance is used in the Yakutsk array as one of the criteria for selecting showers for further analysis and obtaining the exact characteristics of an EAS.
\end{abstract}

35th International Cosmic Ray Conference - ICRC2017-

12-20 July, 2017

Bexco, Busan, Korea

* Speaker.

${ }^{\dagger}$ A footnote may follow. 


\section{Introduction}

When carrying out precision optical measurements, which include the measurement of the Vavilov-Cherenkov light flux from extensive air showers (EAS), it is required to know the characteristics of the medium (i.e., the air density in height and the function of atmospheric transmission) in which the radiation propagates. This is important for determining the main parameters of the development of the EAS in height, i.e. Cascade curve [1] and an estimate of the shower energy based on the total flux of Cherenkov light EAS measured at sea level [2].

\section{Experiment and calculations}

The basis for such an analysis was the observations of the Cherenkov light of the EAS under different weather conditions. Gradations of weather conditions and their binding to spectral transparency are given in [3]. Observations showed that the lateral distribution function, namely, its slope changes, if we consider excellent weather conditions (score 5 points) and subsequent gradations, 4, 3 and 2 points [4]. In describing the experimental data and comparing them with similar measurements in other installations, the terminology adopted in meteorology is usually used. Therefore, in order to establish the correspondence between the ball system and the visibility range $\mathrm{V}$, calculations have been made that take into account the transparency of the atmosphere and its influence on the formation of the spatial distribution of the Cherenkov light of the EAS at sea level [5]. Below we describe the calculations and compare them with the experimental data.

\section{1 $T(z)$ function calculations for different models of the atmosphere}

In paper [6], using the LOWTRAN7 program [7], transmission coefficients T (z) were calculated, taking into account different sets of the atmospheric model. In order to select a suitable model of the winter atmosphere, the calculated functions $\mathrm{T}(\mathrm{z})$ were compared with the experimentally found transmission coefficients $\mathrm{Te}(\mathrm{z})$ of the atmosphere over the Yakutsk array in meteorological states (transparencies) of the atmosphere in 3, 4 and 5 Points.

It turned out that it is possible to obtain the agreement of the functions $\mathrm{T}(\mathrm{z})$ with the functions $\mathrm{Te}(\mathrm{z})$ to the accuracy of the experimental data by selecting the meteorological visibility range of $\mathrm{V}$ - the input parameter that affects the aerosol concentration in Boundary layer $(0-2 \mathrm{~km})$ for a winter subarctic cloudless atmosphere and a rural aerosol, in the absence of an aerosol of volcanic origin. It was found that the transparency of 5 points can be put in correspondence $\mathrm{V}=22 \mathrm{~km}, 4$ points $\mathrm{V}=15 \mathrm{~km}, 3$ points $-\mathrm{V}=10 \mathrm{~km}$. An example of a comparison of $\mathrm{T}(\mathrm{z})$ and $\mathrm{Te}(\mathrm{z})$ is shown in Fig. 1 .

\subsection{Calculations of Cherenkov light flux.}

The radial distribution of Cherenkov radiation was calculated by numerical solution of the system of conjugate cascade equations in the small-angle approximation [6]. At the same time, the transmittance coefficients of the atmosphere program were used [7]. The transmittance coefficients calculated using the LOWTRAN 7.

The calculations were performed for an electron-photon cascade induced by a primary photon with an energy of $10^{18} \mathrm{eV}$, which appeared at the boundary of the troposphere. The calculations 


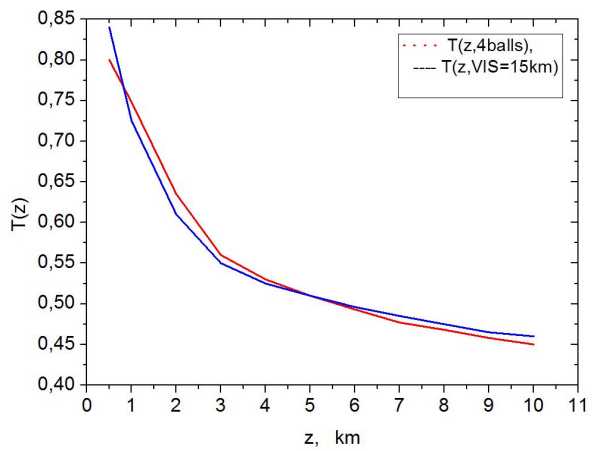

Figure 1: Coefficients for atmospheric transparency 4 points and visibility range $V=15 \mathrm{~km}$.

were carried out for different grades of the visibility range $\mathrm{V}$, which were close to the conditions for carrying out measurements of the Cherenkov light of the EAS at the Yakutsk array [4].

\section{Transparency of the atmosphere influence to measurement of Cherenkov light flux density}

In this case, individual functions of the lateral distribution of Cherenkov light (LDFCL) in showers with energies of $5 \cdot 10^{17}-5 \cdot 10^{18} \mathrm{eV}$, selected according to weather conditions are considered: excellent weather ( 5 points), good weather (4 points) and satisfactory weather (3 points ) [4]. All showers were normalized to an energy of $10^{18} \mathrm{eV}$ and the local flux densities of the Cherenkov light EAS were averaged over narrow distances. Next, the ratio of the Cherenkov light flux EAS for different gradations of weather conditions $(5 / 4,5 / 3$ and 4/3) was considered in the case of experimental data and similarly for the calculated LDF CR. The results of the comparison are shown in Fig. 2, Fig.3 and Fig. 4.

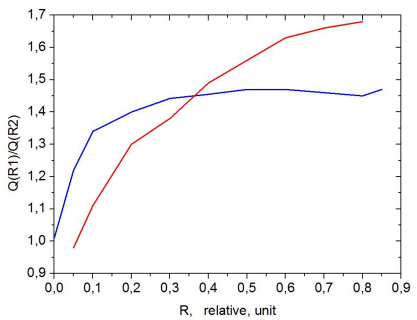

Figure 2: Ratio of Cherenkov photons flux in showers produced by primary gammaray at visibility $\mathrm{V} 22 \& 10 \mathrm{~km}$ (calculations, blue curve). The ratio of flux density of Cherenkov photons with proton as primary (red dashes) for the atmospheric transparency $5 \& 3$ points âĂŞ experimental data [4].

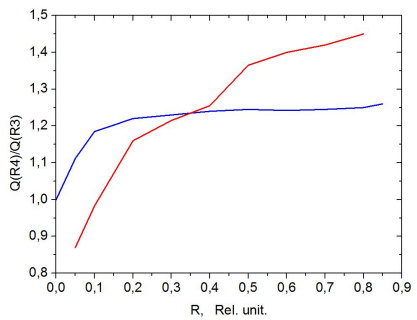

Figure 3: Ratio of Cherenkov photons flux in showers produced by primary gammaray at visibility $\mathrm{V} 15 \& 10 \mathrm{~km}$ (calculations, blue curve). The ratio of flux density of Cherenkov photons with proton as primary (red dashes) for the atmospheric transparency $4 \& 3$ points âĂŞ experimental data [4] 


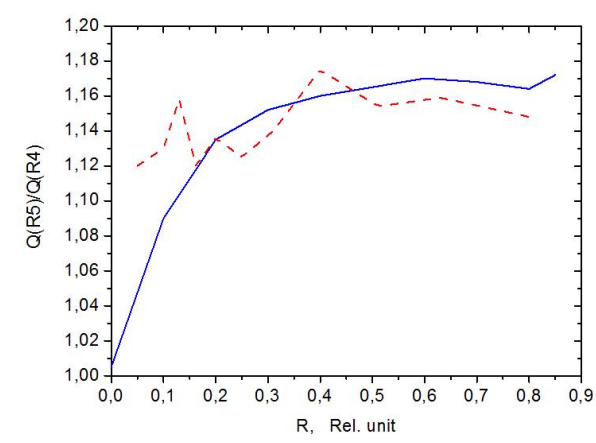

Figure 4: Ratio of Cherenkov photons flux in showers produced by primary gamma-ray at visibility V 22 $\& 15 \mathrm{~km}$ (calculations, blue curve). The ratio of flux density of Cherenkov photons with proton as primary (red dashes) for the atmospheric transparency $5 \& 4$ points - experimental data [4].

\section{Conclusion}

Recently, due to warming [8,9], in the winter period at the Yakutsk array area there is a frequent alternation of cyclones and anticyclones, which create not favorable conditions for observing Cherenkov light of EAS in the visible wavelength range (350-800) $\mathrm{nm}$. Under these conditions, much attention must be paid to observations of the state of the atmosphere in order to obtain information on the transparency of the atmosphere and quantitative estimates of the transmittance function of the atmosphere Te $(\lambda)$. Comparison of the experimental data (Fig. 2 and Fig. 3) and calculations showed that with a visibility range $\mathrm{V}<10 \mathrm{~km}$, a large concentration of different types of aerosol in the atmosphere (ground haze, fog, low clouds) is assumed, which leads to a distortion of the shape of the spatial light function of the EAS.

\section{Acknowledgments}

The reported study was funded by RFBR according to the research project 16-29-13019.

\section{References}

[1] S.P. Knurenko et al. Proc. 27th ICRC. Hamburg. 1, 157 (2001)

[2] S.P. Knurenko, A.A. Ivanov, I.Ye. Sleptsov, A.V. Sabourov, JETP Lett. 83, No. 11, 563-567 (2006).

[3] S.P. Knurenko, S.V. Nikolashkin, A.V. Saburov, I.Ye. Sleptsov. Proc. Of SPIE. 6522 (2006).

[4] M.N. Dyakonov, S.P. Knurenko, V.A. Kolosov, I.Ye. Sleptsov. Optics of the atmosphere and ocean. 12, 329-333 (1999).

[5] M.N. Dyakonov, S.P. Knurenko, V.A. Kolosov, I.Ye. Sleptsov. Optics of the atmosphere and ocean. 4, 868-873 (1991).

[6] A. I. Goncharov, N.G. Nikitenko, Bulletin of AGU, 85-88 (2004).

[7] F.X. Kneizys, E.P. Shettle, L.W. Abrew et al. Scientific report of Air Force Geophysics Laboratory AFGL-TR-88-0177. Hanscom, 146, (1988) 
[8] S.P. Knurenko, V.A. Kolosov, I.Ye. Sleptsov. Proc. of 9th International Symposium Tomks, 348 (2002).

[9] S.P. Knurenko, I.S. Petrov. Proc. of XIX Intern. Symp. âĂIJOptics of the atmosphere and ocean. Physics of the atmosphereâĂI. D357-D360 (2013) 